\title{
Role of FDG-PET/CT in prediction of pathological tumor response and survival after trimodality therapy for esophageal squamous cell carcinoma
}

\author{
Yoichi Hamai", Jun Hihara, Takaoki Furukawa, Ichiko Yamakita, Tomoaki Kurokawa, Morihito Okada \\ From World Society of Cardiothoracic Surgeons 25th Anniversary Congress, Edinburgh \\ Edinburgh, UK. 19-22 September 2015
}

\section{Background/Introduction}

Trimodality therapy has frequently been applied to patients with locally advanced esophageal cancer. However, to preoperatively predict the response to neoadjuvant therapy and prognosis is difficult.

\section{Aims/Objectives}

The present study aimed to determine the diagnostic applicability of $18 \mathrm{~F}$-fluorodeoxyglucose-positron emission tomography/computed tomography (FDG-PET/CT) to evaluating the response and prognosis after trimodality therapy for esophageal squamous cell carcinoma (ESCC).

\section{Method}

The responses of 111 patients with ESCC who underwent neoadjuvant chemoradiotherapy (nCRT) followed by surgery were monitored by measuring the maximal standardized uptake value (SUVmax) of the primary tumor on FDG-PET before and after nCRT. Associations between SUVmax and pathological response of primary tumor and prognosis were analyzed.

\section{Results}

We compared the SUVmax between good (Japan Esophageal Society response evaluation criteria grades $3 / 2$; $\mathrm{n}=87,78.4 \%$ ) and poor (grade $1 ; \mathrm{n}=24,21.6 \%$ ) responders. The SUVmax after nCRT (post-SUVmax) in good and poor responders were $2.7 \pm 0.9$ and $4.4 \pm 2.2$, respectively $(\mathrm{p}<0.0001)$. The rates of the SUVmax decrease after nCRT $(\triangle S U V \max )$ in these patients significantly differed $(71 \pm 14 \%$ vs. $60 \pm 21 \%$, respectively, $p=0.003)$. The area under receiver operating characteristic curves (AUC) showed that the optimal cut-off for post-SUVmax and $\triangle$ SUVmax were 3.7 (AUC, 0.76; 95\% CI, 0.63-0.89; $\mathrm{P}<0.001$ ) and 70\% (AUC, 0.65; 95\% CI, $0.52-0.78 ; \mathrm{P}=0.02$ ) for predicting good responder, respectively, and that the patients could be separated into groups with and without good response using these cut-off values. The 5-year overall survival rate was significantly higher for patients with $\triangle$ SUVmax $>70 \%$ than $\geq 70 \%(66 \%$ vs. $42 \%, p=0.04)$, and multivariable analysis including preoperative factors also revealed $\triangle \mathrm{SUV}$ $\max (\geq 70 />70 \%)$ as an independent prognostic factor for disease-specific survival (OR, 2.22; 95\%CI, 1.02 $4.76 ; \mathrm{p}=0.04)$.

\section{Discussion/Conclusion}

Post-SUVmax and $\triangle$ SUVmax were valuable preoperative factors for predicting tumor response and survival in patients with ESCC after trimodality therapy. Thus, FDG-PET/CT findings are useful for tailoring optimal therapies for individual patients with ESCC.

Published: 16 December 2015

doi:10.1186/1749-8090-10-S1-A244

Cite this article as: Hamai et al:: Role of FDG-PET/CT in prediction of pathological tumor response and survival after trimodality therapy for esophageal squamous cell carcinoma. Journal of Cardiothoracic Surgery 2015 10(Suppl 1):A244 